\title{
Silver Nanoparticles Plasmonic Response in the Proximity of an Interface
}

\author{
Gursoy B Akguc* \\ Department of Physics, Izmir University of economics, Turkey
}

\begin{abstract}
Silver nanoparticles near other silver nanoparticles or near a substrate have modified plasmonic resonances which maybe important in device applications. Having plasmonic solar cell applications in mind, various aspect of silver nanoparticles on transparent conducting oxide (TCO) thin film structures are investigated. The shift in resonance has been calculated for the different shape, size and, distance of silver particles on various refractive index of the TCO. The effect of inter nanoparticle effects are discussed as well. The plasmonic resonance curve may change in case of inhomogeneity in silver nanoparticles geometry as well as proximity to each other.
\end{abstract}

PACS numbers: 72.20.Dp, 72.20.Pa, 73.21. Hb, 73.23.Ad, 73.63.Nm

\section{Introduction}

In device application, such as in plasmonic solar cells, it is common to utilize silver as an agent to interact with the long wavelength part (near infrared) of the sun spectrum which can be archived through plasmonic resonance [1].

There is no unique plasmonic response of nanoparticles, on the contrary, a plethora of ways to manipulate them. When there is no inhomogeneity in the system, the shapes and sizes of nanoparticles as well as the homogenous medium they are in seem to be the important parameters [2-5]. More interestingly when there are inhomogeneities in the system, as in the most practical cases, interaction due to interfaces become important $[6,7]$. The interaction of nanoparticles become important in other applications like plasmonic detectors, antennas where one can use electromagnetically induced transparency [8].

We solve full three dimensional (3D) time dependent electromagnetic equation using finite difference time domain method (FDTD) [9] in order to look at various configuration of metallic nanoparticles with respect to TCO film. FDTD is being used recently in plasmonic calculation of complex systems $[7,10]$. When silver nanoparticles approach to the TCO film their plasmonic behavior changes as a function of their distance from the substrate as expected. When it is close enough we observe a new dipole excitation in the system which is red shifted than the one get in homogeneous system. We look at the effect of film thick-ness and the relative locations of nanoparticles with respect to TCO. We observe that we can manipulate the coupling to the TCO by changing the shape of nanoparticles. The film thickness seems to be a relevant parameter in the manipulation of this new red shifted dipole resonances $[11,12]$.
We use boundary element method BEM solver [13] for the effect of size and shape averaging and interaction among $\mathrm{Ag}$ nanoparticles which should be taken into account in explaining experimental result. We show that interaction among nanoparticles causes red shifted dipole resonances [14]. Also averaging should not be thought as a linear process by showing size matters to define the plasmon resonance.

In following section we discuss nanoparticle-tco interface and FDTD method we use. In section III We look into interaction of nanoparticles by themselves where we utilized BEM in calculations. We conclude in section IV.

\section{Plasmon Response due to Interaction of Nanoparticle with TCO Thin Film}

Plasmon resonance of $\mathrm{Ag}$ nanospheres inside homogeneous medium can be calculated exactly using mie theory. Extension to spheroids also possible [2]. In Figure 1 we show plasmon behavior of $\mathrm{Ag}$ nanoparticles inside a homogeneous medium as a reference for the interaction case. In Figure 1a we show effect of shape by changing one of the principal axis of sphere. As the shape goes from sphere to a prolate shape plasmon resonance red shift. In Figure $1 \mathrm{~b}$ we show size dependence. Scattering cross section is only effective when pass

*Corresponding author: Gursoy B Akguc, Department of Physics, Izmir University of economics, 35330, Izmir, Turkey

Accepted: August 07, 2019

Published online: August 09, 2019

Citation: Akguc GB (2019) Silver Nanoparticles Plasmonic Response in the Proximity of an Interface. Aspects Nanotechnol 2(1):61-69

Copyright: (C) 2019 Akguc GB. This is an open-access article distributed under the terms of the Creative Commons Attribution License, which permits unrestricted use, distribution, and reproduction in any medium, provided the original author and source are credited. 


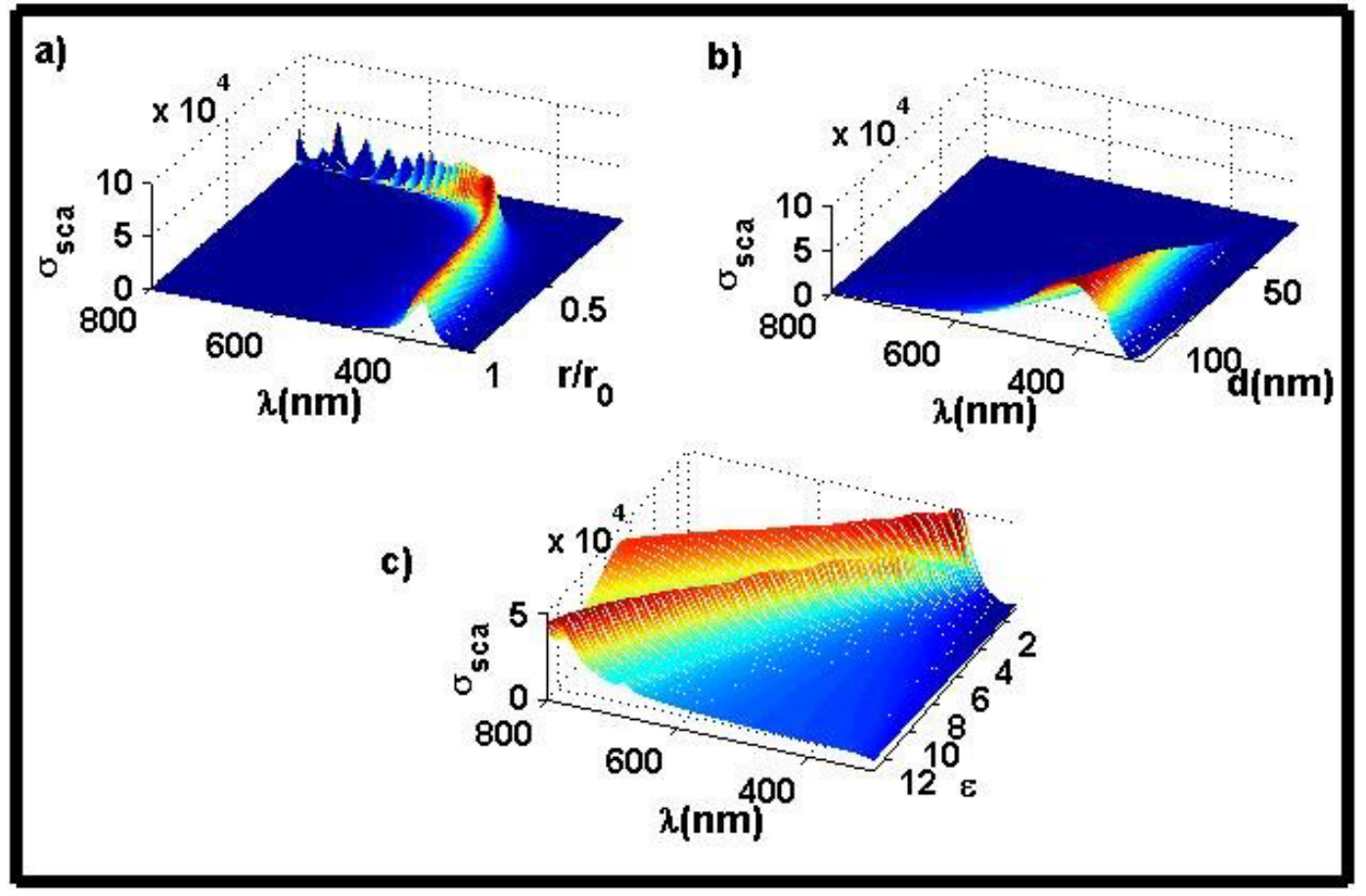

Figure 1: (Color online). Plasmonics in homogenous medium. a) Spheroid with base sphere of $r_{0}=50 \mathrm{~nm}$; b) Spheres with changing diameter; c) A reference sphere of radius $r_{0}=50 \mathrm{~nm}$ in a homogeneous medium with changing refractive index.

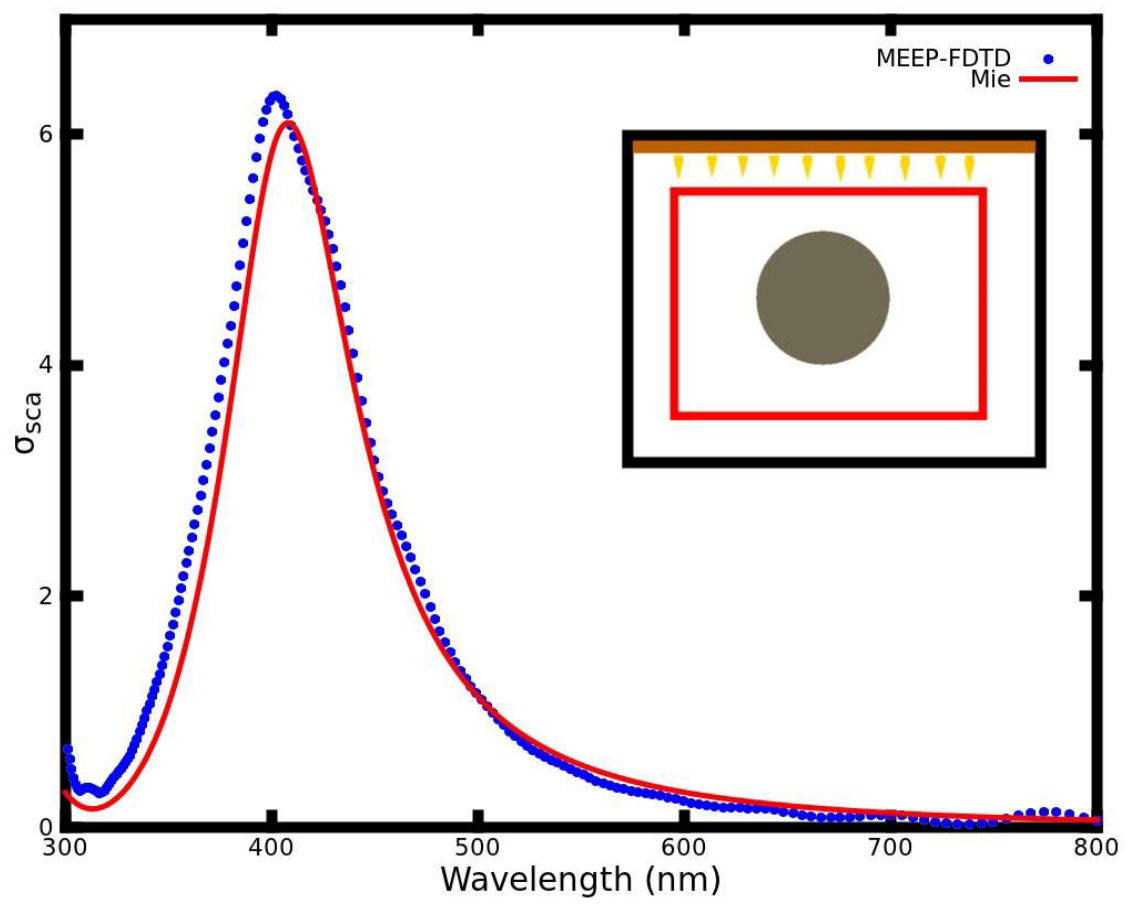

Figure 2: (Color online). Ag nanosphere in vacuum.

a critical radius, at around $\mathrm{D}=70 \mathrm{~nm}$ scattering cross section becomes dominant. For small radius spheres absorption inside sphere is prevalent and not useful in solar cell application. In Figure 1c we show the effect of homogenous medium. As the dielectric constant of medium increases plasmon frequency red shifts and higher order modes, i.e. quadrupole, octupole moments appears.
More interesting is the behaviour of $\mathrm{Ag}$ nanoparticles in inhomogeneous medium. In almost all cases we want nanoparticles near or inside a semiconductor interface. This is an important design parameter for solar cell application. Due to large surfaces involved a BEM approach is not practical here that is why we use FDTD method to solve the electromagnetic wave equation. We give some details about the method in following. 


\section{FDTD, FEM and BEM methods}

We use finite discrete time domain (FDTD), finite element method (FEM) and Boundary element method (BEM) to solve Maxwell's equations numerically. We give brief description in this section for various methods we use in this work.

In it's basic understanding FDTD is the method that Maxwell's equations are evolved in discretized grid of space and time.

To simulate silver nanoparticle we use an approximation to the experimentally available dielectric constant of silver in terms of a Drude-Lorentz series as in the litearature [15]. We show a sample calculation in Figure 2 with the inset describing geometric boundaries and the imaginary surfaces to calculate the scattering cross section. A radius $45 \mathrm{~nm}$ sphere in air has a scattering cross section of light as shown both with FDTD result and mie exact result.

We have a computational region of $600 \times 600 \times 600 \mathrm{~nm}^{3}$ shown as the outermost region. We need to use perfect matching layers as boundary conditions so $100 \mathrm{~nm}$ thick reflectionless region is set at the outermost region. Than we choose an imaginary surface, a cube of size $350 \times 350 \times$ $350 \mathrm{~nm}^{3}$ (shown as red line), to calculate the scattering cross section. Source is just at the edge of PLM region and sends gaussian wave. We propagate the electromagnetic wave in this system and in the auxiliary one where there is no sphere so that we can look

at the change in flux. The difference of Poynting vectors, $P(\omega)$ which is defined by $I$

$$
P(\omega)=\operatorname{Re\hat {n}} \cdot \oint_{\omega}(x)^{*} \times \mathrm{H}_{\omega}(x) \mathrm{da}
$$

is then used to calculate the scattering cross section by,

$$
\sigma_{s c a}(\omega)=\frac{P(\omega)}{I(\omega)}
$$

Where $\hat{n}$ is the outward normal direction to the surface, $I(\omega)$ is the intensity of light.

In following plots scattering cross section efficiency is shown where we divide the scattering cross section to the geometric cross section.

We use a typical computational cell size of $1.25 \mathrm{~nm} \times 1.25$ $\mathrm{nm} \times 1.25 \mathrm{~nm}$ to get the accuracy shown in Figure 2. We run parallel MEEP and a typical run for any system takes about 10 hours CPU in a $4 \times 8$ AMD core PC system.

\section{Interaction with TCO}

We look into the effect of nanoparticle-TCO interface on plasmonic response. We use an FDTD method to find the scattering cross section efficiency as described earlier. Only addition is a semiconductor region of infinite thin film with varying thickness in to calculation. We choose a typical TCO thin film of indium titanium oxide (ITO) to demonstrate the effect of silver nanoparticle interaction with substrate in Figure 3. As we lower the silver sphere of radius, $R=30 \mathrm{~nm}$ on top of the ITO thin film we see a new resonance peak appears in total scattering cross section efficiency. The amplitude of red shifted dipole resonance increases when silver nanparticle approaches to the film. We use a film thickness of $t=$ $200 \mathrm{~nm}$ used in Figure 4a. We also observe a red shif in the position of the original dipole moment as it gets closer to the surface. In Figure $4 \mathrm{~b}$ we observe similar effects for a spheroid. But note that in this case actually the intensity of second peak is much higher than the original dipole resonance peak, practically making big change in response of the system. We note that the place of red shifted dipole resonance is comparable with the dipole resonance of a homogeneous system of silver sphere inside an ITO medium. In Figure $4 c$ and Figure $4 d$ we show the effect of thickness of ITO film on plasmonic response. In both cases silver sphere and spheroid stays on top of ITO film. We see that a blue shift of new resonance dipole position with decreasing thickness. In Figure $4 d$ we see this blue shift effects the intensity of original dipole resonance

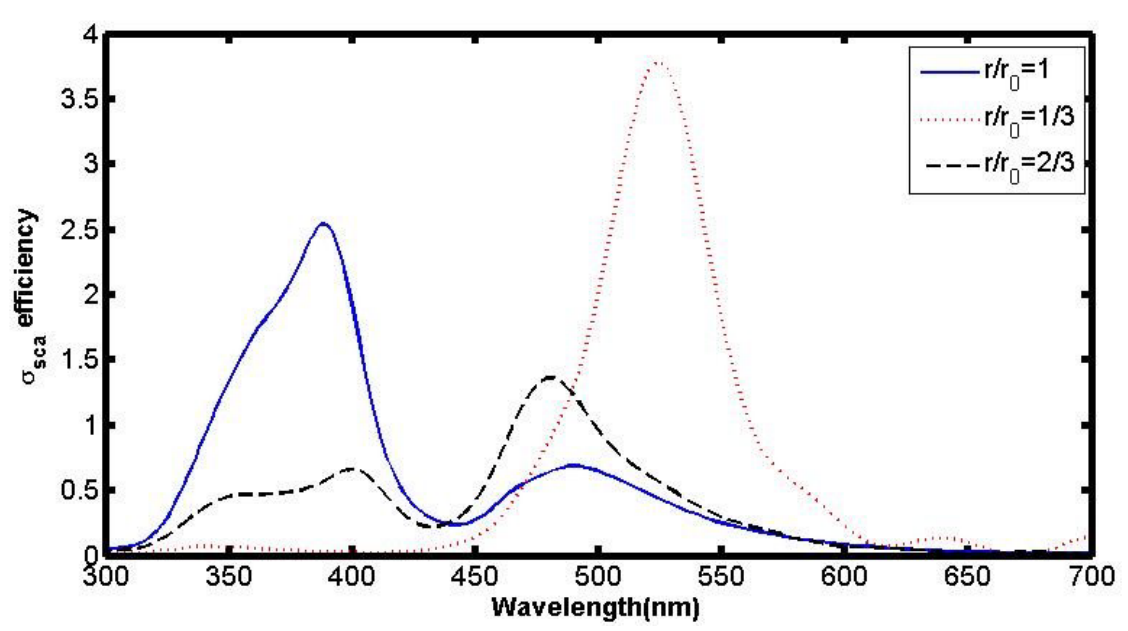

Figure 3: (Color online). a) Silver sphere at different heights on top of ITO film of thickness $t=200 \mathrm{~nm} ; \mathrm{b}$ ) Silver spheroid of ratio $r / r_{0}=2 / 3$ at different heights on top of ITO film; c) Silver sphere with various thickness of ITO film; d) Silver spheroid of ratio $r / r_{0}=2 / 3$ with various thickness of ITO film. In all plots a reference sphere of radius, ${ }_{0}=30 \mathrm{~nm}$ is used. 

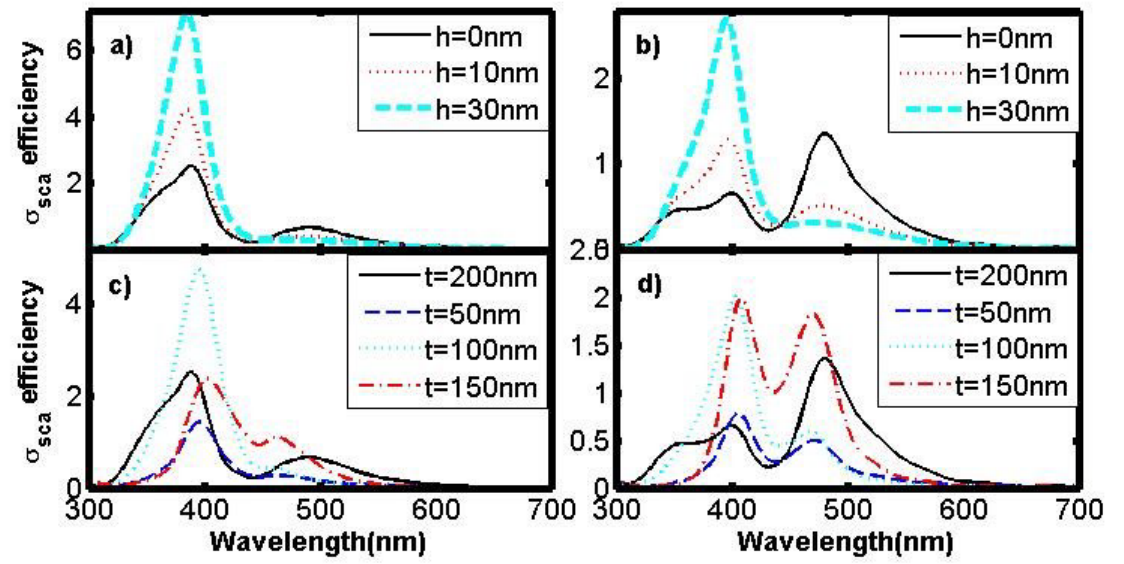

Figure 4: (Color online). Spheroid on ITO thin film for a reference silver sphere of radius, $r_{0}=30 \mathrm{~nm}$.

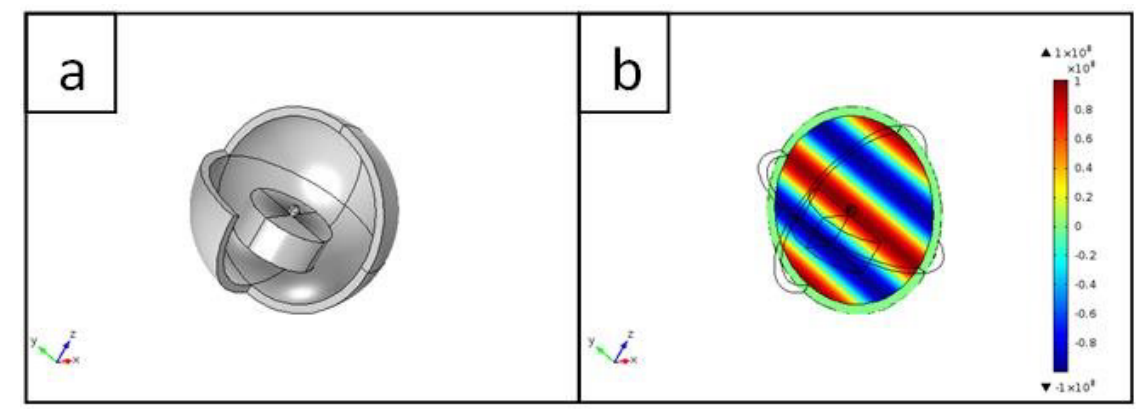

Figure 5: (Color online). a) Typical geometry used in FEM calculations; b) The incident plane wave of = 500 is shown on $y z$ working plane.

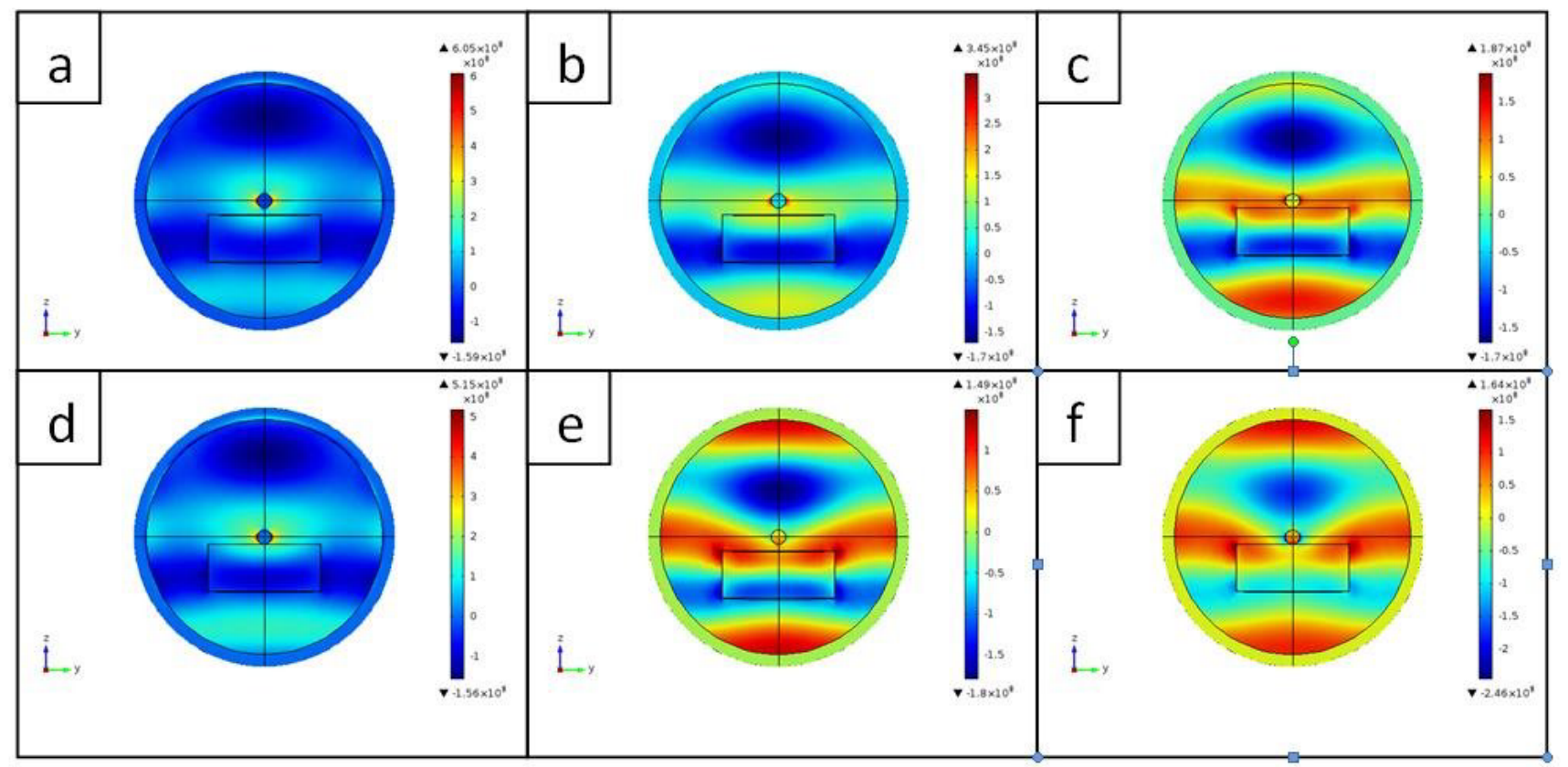

Figure 6: (Color online). Sphere $30 \mathrm{~nm}$ above the ITO substrate and with the radius $30 \mathrm{~nm}$ interacts with incoming plane wave of wavelength a) $400 \mathrm{~nm}$; b) $450 \mathrm{~nm}$; c) $500 \mathrm{~nm}$ some for the sphere touching to the surface incident by the wavelength; d) $\lambda=400 \mathrm{~nm}$; e) $\lambda=450 \mathrm{~nm}$; f) $\lambda=500 \mathrm{~nm}$.

and it becomes almost similar in size. It is possible to change the intensity of resonance peak by changing film thickness.
We find that it is possible to manipulate the intensity of resonance peaks so making effectively coupling to substrate 
something adjustable by changing the prinsipal axis of spheroidal silver nanoparticles. In Figure 3, we show by making a prolate shape sphere we can increase the intensity of redshifted dipole resonance. In practice prolate spheroidical shape is more common.

We have also used Finite element method to compare our results from FDTD as well as to see specifically the excitations for the resonance wavelengths.

In FEM, we discretize scattering region with finite polynomials and solve weak form of maxwells equation with appropriate boundary conditions. A typical geometry is shown in Figure 5.

In Figure 6, we show the plasmonic excitation correspond to the resonance for spherical silver shapes as found previously in Figure 4 using FDTD. The magnitude of electric field before, on and, after the resonance shown for silver spheres away from the TCO and on the TCO respectively. Here we chose to show the magnitude of y component of electric field. The corresponding colorbar is also shown in figures. We look at the response of the sphere to incoming light with various wavelength chosen from the figure. general at 3 points before on and after resonance.

In Figure 7, plasmonic excitations before on and after resonance for prolate silver nanoparticles are shown.

In Figure 8, we show at resonance and out of resonance case of a prolate on TCO in close up.

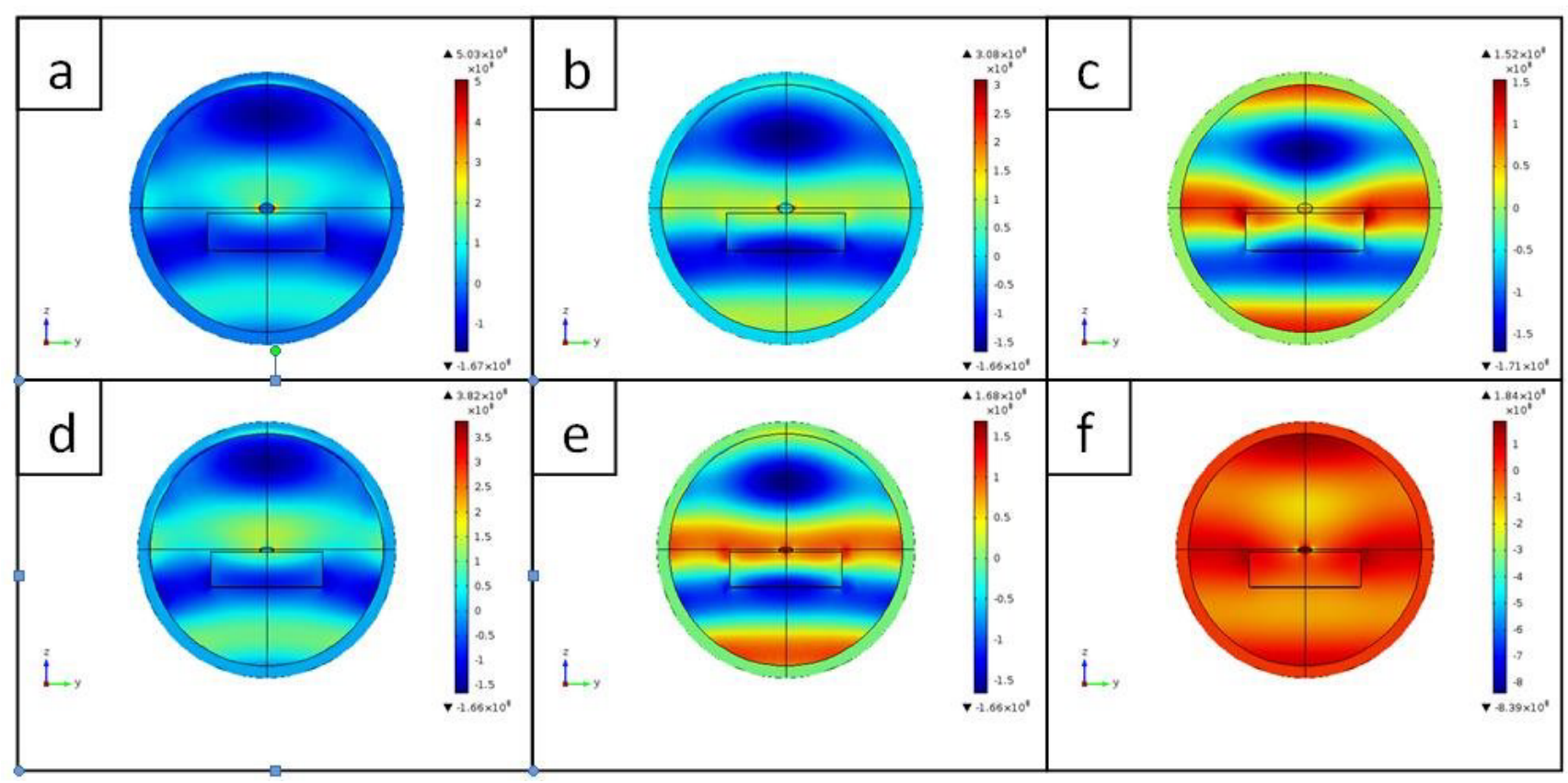

Figure 7: (Color online). An ellipsoid with principal semiaxis, $a=30, b=30, c=20$ is touching on ITO surface with thickness $t=150 \mathrm{~nm}$ is incident upon by a plane wave with wavelengths a) $\lambda=400 \mathrm{~nm}$; b) $\lambda=450 \mathrm{~nm}$; c) $\lambda=490 \mathrm{~nm}$. The principal axis of ellipsoid is reduced further to $c=10 \mathrm{~nm}$ and excited by d) $\lambda=400 \mathrm{~nm}$; e) $\lambda=450 \mathrm{~nm}$; f) $\lambda=540 \mathrm{~nm}$.
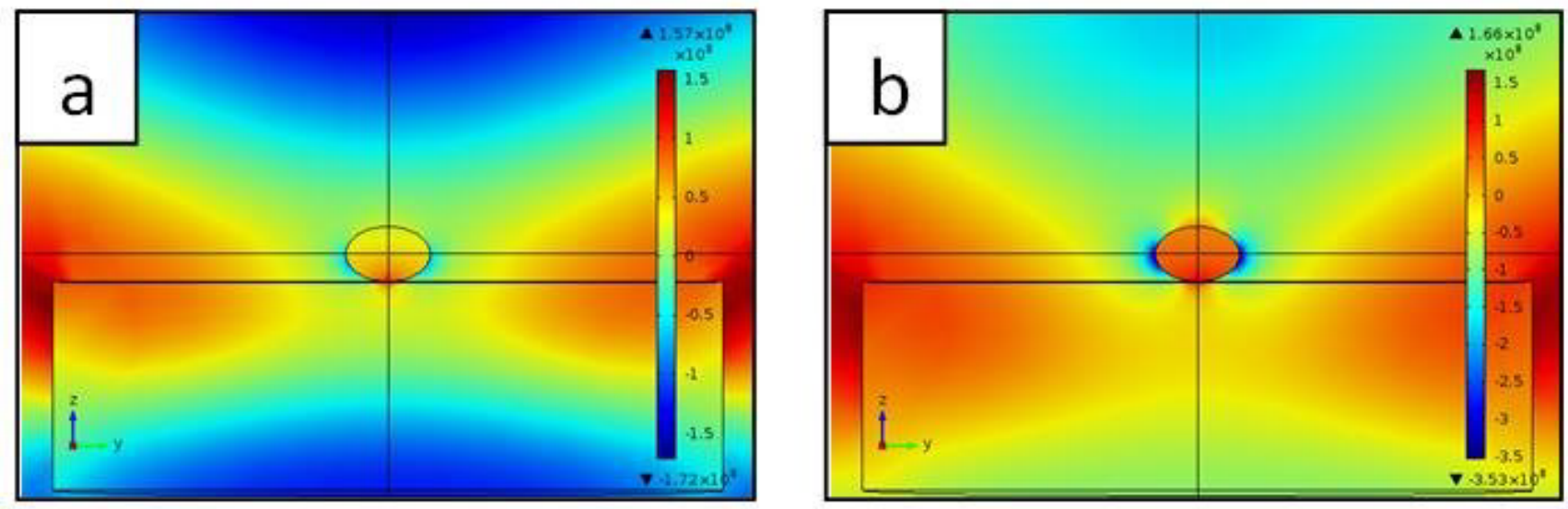

Figure 8: (Color online). The y component of Electric field near the second scattering peak identified by FDTD calculations is shown for the ellipsoid $a=30 \mathrm{~nm} ; b=30 \mathrm{~nm} ; c=20 \mathrm{~nm}$ in a) just before resonance $\lambda=500 \mathrm{~nm}$ and b) at resonance $\lambda=540 \mathrm{~nm}$. 


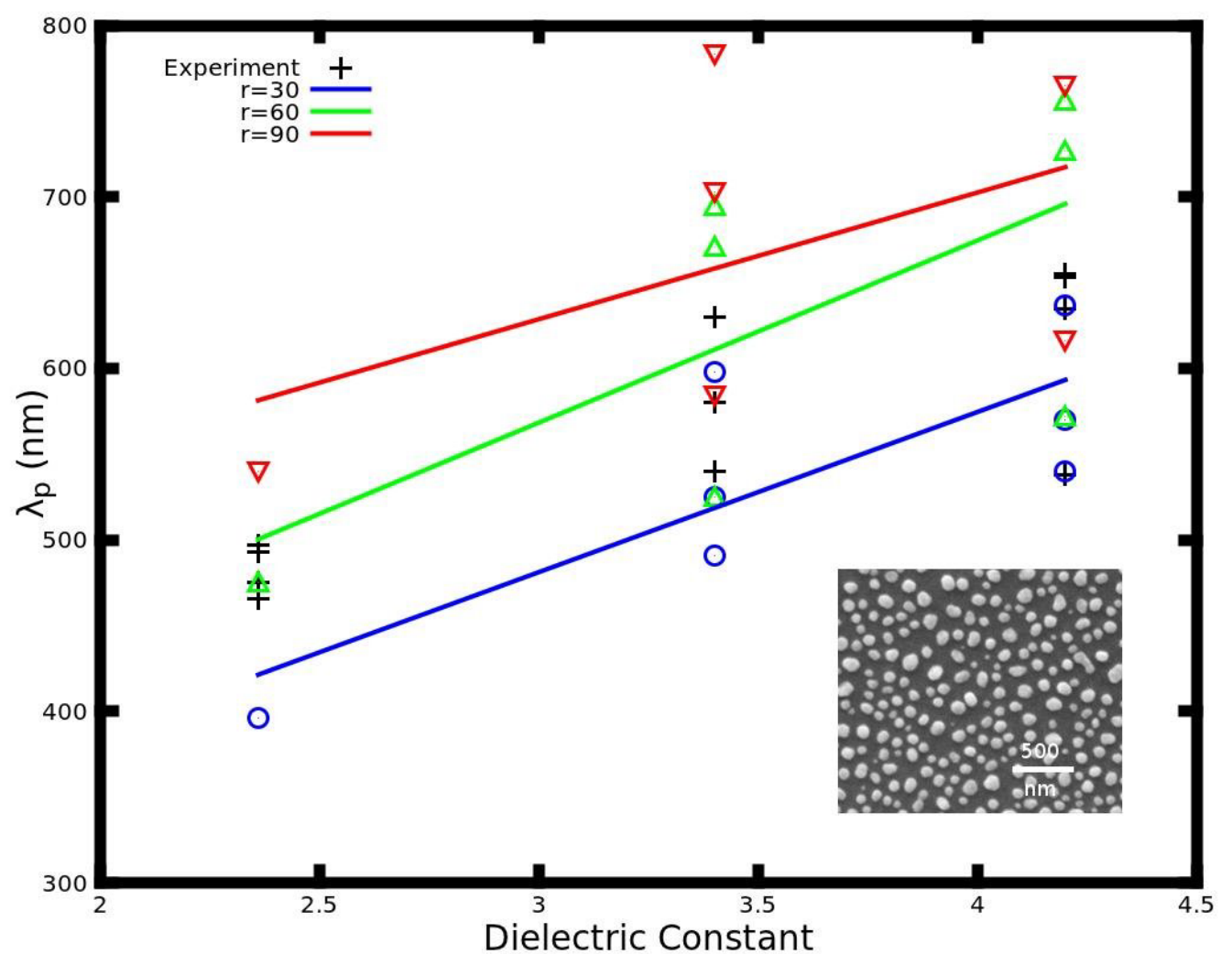

Figure 9: (Color online). Experimental and theoretical plasmon resonance changes as a function of dielectric constant. Inset show a experimental picture of silver nanoparticles on top of ITO.

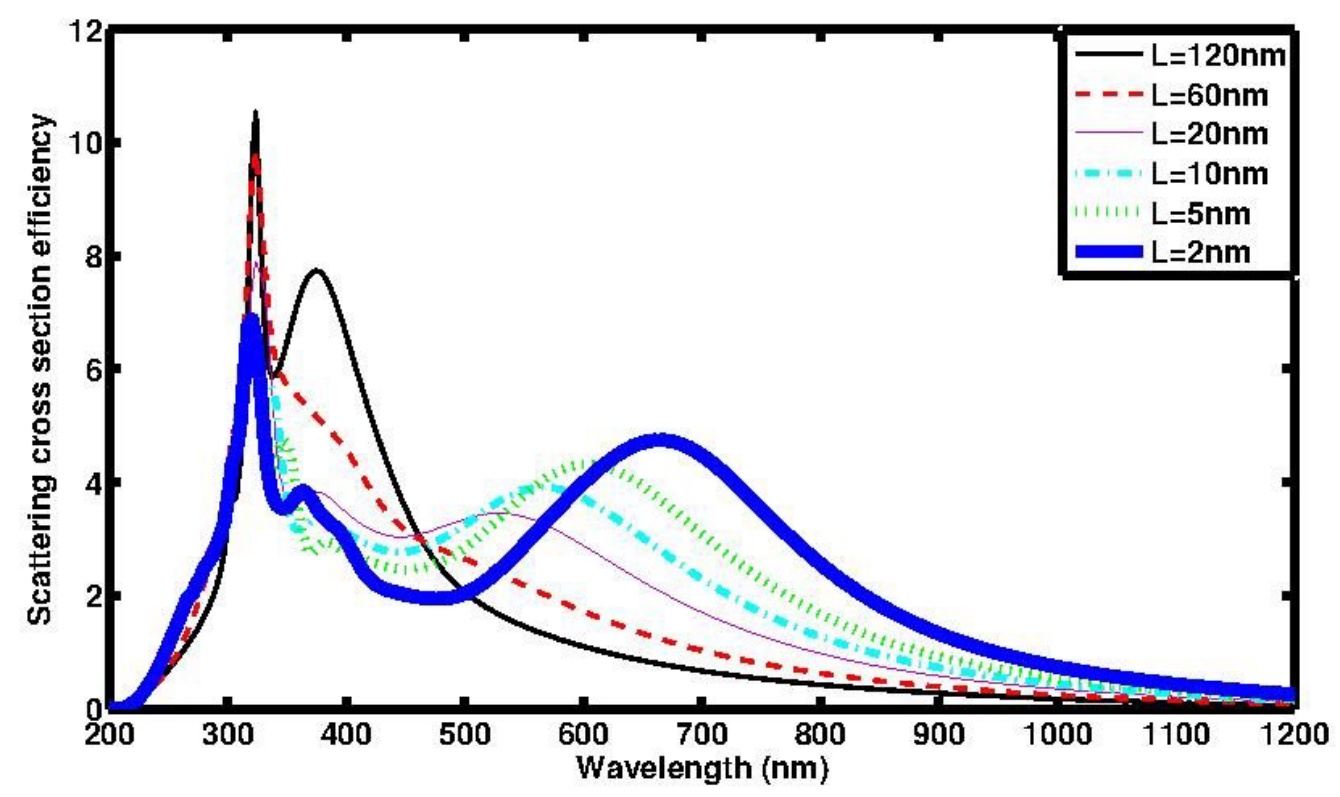

Figure 10: (Color online). Scattering cross section of silver nanoparticles with same size packed with varying proximity.

We also look at the sphere dipped into substrate, half sphere on top of substrate and more to mimic the experimental findings. In Figure 9 we show these simulations along with the experiment as depicted in inset.

For better solar cell design the effect of substrate should be taken into account. Another important interaction is nanoparticles with their neighbors which we discuss next.

Plasmon Response due to Interaction of Nanoparticle with Neighbors

In this section we discuss other important inhomogeneity, i.e. two metallic particles come close to each other. In exper- 
iments that is always the case. Although about 10 percent coverage of solar cell is enough to scatter all incoming light due to the scattering efficiency of nanoparticles is about ten, there is no homogeneity in distribution of particles in practice. There maybe few particles come to close each other and start to interact. There is also issue of averaging cross section when interaction is present. Linear averaging is not the right answer most of the time.

In Figure 10, we show the effect of interaction on the plasmon resonance for $\mathrm{Ag}$ nanospheres in air. To make it polarization independent we use a unit cell of 3 equal size spheres in calculation. The distance $L$ between spheres in $\mathrm{x}$ and $y$ direction is changed same amount in the figure. We see that when two spheres come close there will be a red shift of dipole resonance while quadrupole resonance remains at its original place. There is a also a reminiscent peak in the place of dipole resonance of single particle. In the following figures we show scattering efficiency as before. Note that the effective geometric cross section is projection of three spheres on $x-y$ plane. When there is no interaction (far apart particles) it is possible to take linear averaging of cross sections but not the cross section efficiency.

In Figure 11, we show the surface charge distribution at the resonance positions for $L=2 \mathrm{~nm}$ case in Figure 10. In Figure 11a we see dipole resonance at $\lambda=664 \mathrm{~nm}$ is no longer due to oscillations of electrons between to ends of a sphere (which is the case for a single sphere at $\lambda=395 \mathrm{~nm}$ ) but actually between two neighboring spheres. Another resonance occur

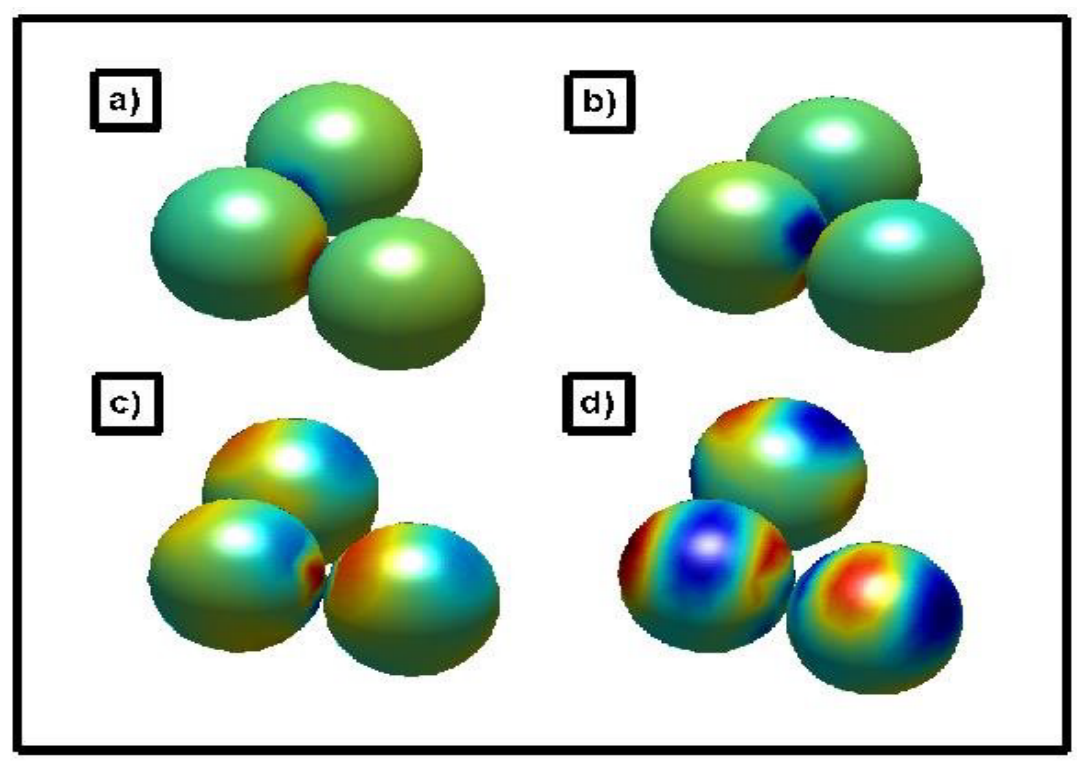

Figure 11: (Color online) Plasmonic excitations of silver spheres with same size when they are closely packed.

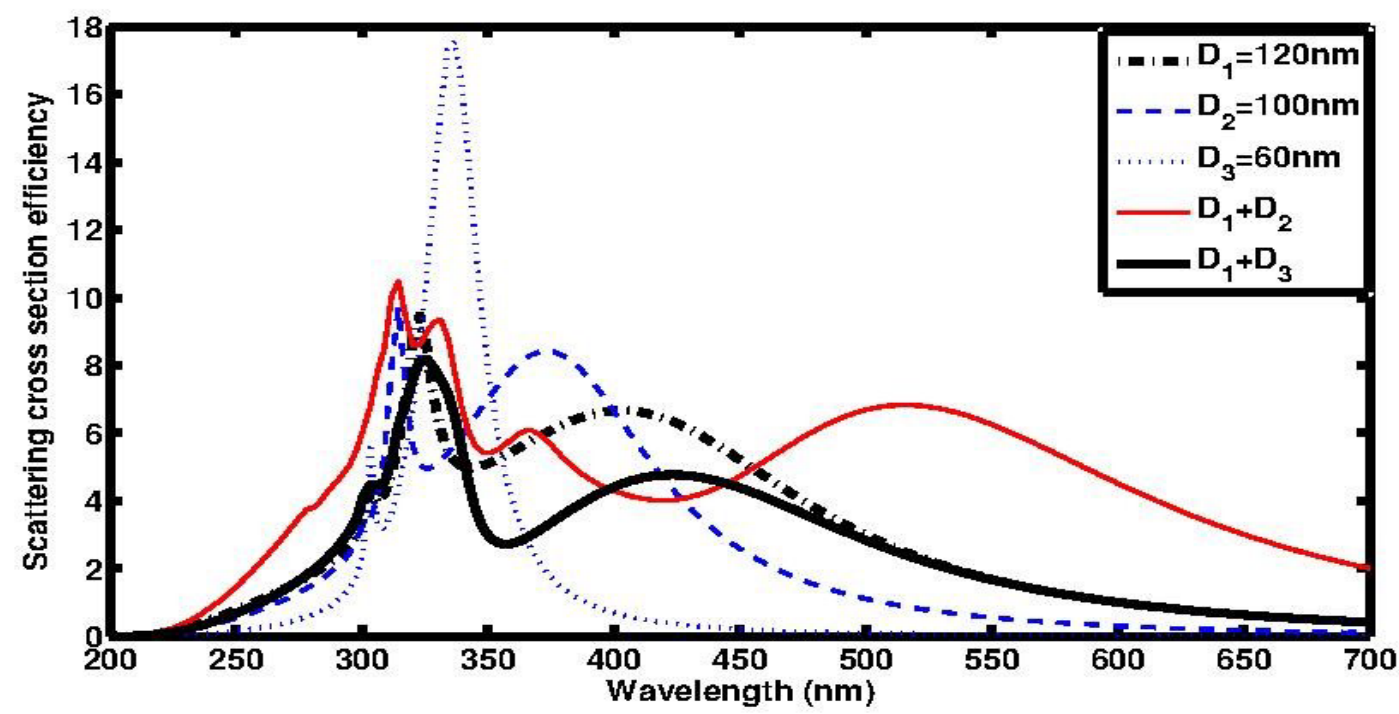

Figure 12: (Color online) Scattering cross sections of three individual silver sphere with various diameters and their pair proximity as shown in legend. 
at $\lambda=324 \mathrm{~nm}$ again between two neighboring spheres in the form of a quadrupole moment as shown in Figure 11b. In Figure $11 \mathrm{c}$ at $=303 \mathrm{~nm}$ we show resonance is quadrupole in nature like a single sphere. In Figure $11 \mathrm{~d}$ octupole moment is also prevalent as in single sphere case.

In experiments a wide variety of sizes and shapes of nanoparticles coexists. Overall response of system is an average of plasmonic resonances of all these particles. Since there is interaction among them, average is not trivial. We look at the size and shape effects on averaging and on overall response of nanoparticles.

In Figure 12 we show 3 different sized spherical Ag nanoparticles in air with close and far separation between them. We start with $D_{1}=120 \mathrm{~nm}$ spherical Ag nanoparticle at the center shown with dot-dashed (black) line in Figure 12. The case with two $D_{2}=100 \mathrm{~nm}$ Ag nanospheres added in $\mathrm{x}$ and y direction are shown with dashed (blue) and solid (red) lines where former shows when particles far apart and later shows when they are closely separated. We see similar dipole resonance shift when the sizes close in comparison as before. We see completely different picture when we have small sized spheres replaces as neighboring nanoparticles shown as dotted (blue) and thick solid (black) lines in the same figure. The dotted (blue) curve shows single sphere cross section. We see the effect of small sized sphere makes just resonance of $D=120 \mathrm{~nm}$ sphere wider than usual but not linear effect of adding cross section together. The overall decrease in size of the dipole resonance is due to dividing cross section with geometrical cross section to find the efficiency. The total cross section of this systems is in reality very little different than one single $D=120 \mathrm{~nm} \mathrm{Ag}$ sphere. We note that the effect of dipole shift still observable if we let spheres comes too close here we use $L=10 \mathrm{~nm}$ as the separation length between $D_{1}=$ $120 \mathrm{~nm}$ sphere and $D=60 \mathrm{~nm}$ spheres.

In Figure 13 we show the effect of shapes on averaging of plasmonic scattering cross section. Stand alone particles of $D$ $=120 \mathrm{~nm}$ sphere and $D=60 \mathrm{~nm}$ prolate shape nanoparticle are shown with dash-dotted (blue) and dashed (blue) curves. Two separation distances $L=30 \mathrm{~nm}$ and $L=180 \mathrm{~nm}$ are shown with solid (black) and thick solid (red) curves respectively. We see that red shift due to shape deformation also effects the averaging. The same characteristic dipole shift also observable here as well as shift in the position of quadrupole resonance due to proximity to the spherical nanoparticle at the center.

\section{Concluding Remarks}

Ag nanoparticles in thin film solar cell design should take in to account various geometrical and interface effects into account. We show the effect of interface between metallic nanoparticles and semiconductor TCO on plasmon resonance response. Due to interface to semiconductor new plasmonic peaks observed in simulations.

We also look at the effect of interaction between nanoparticles. While a characteristic red shift in dipole resonance is observed when particles close to each other we see quadrupole resonance does not shift at all. The effect of size on averaging should be also taken carefully since smaller particles has little effect on position of large particles resonance peaks just make their resonance wider.

We had to keep parameter space small to demonstrate our conclusion. There are plenty of other effect comes to play when interaction of nanoparticles, such as humidity of air, medium of interaction, silver dioxide formation [16]. The main interaction effects would persist but renormalized acordingly depending on the permitivity of the transmitting medium between nanoparticles.

\section{Acknowledgments}

We acknowledge support from the Scientific and Tech-

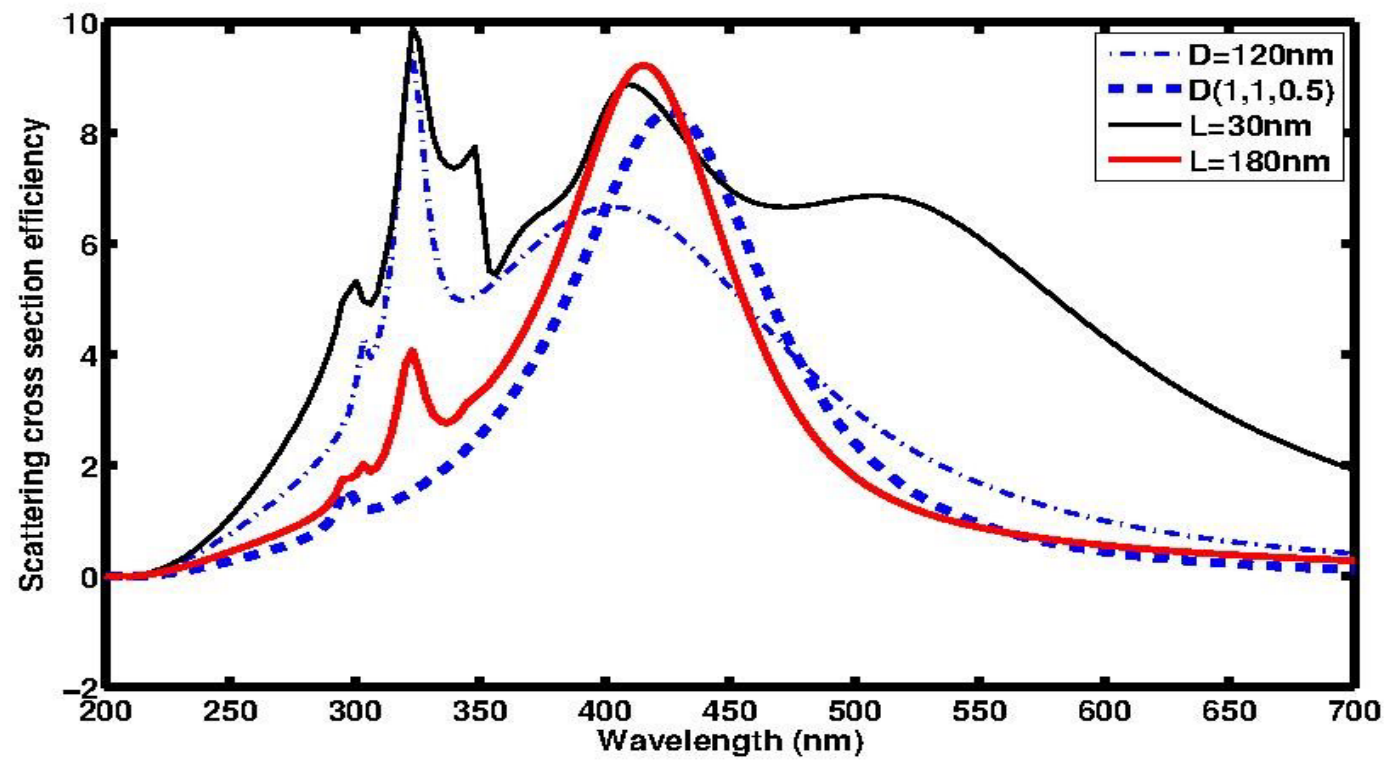

Figure 13: (Color online) Scattering cross section of spherical and prolate shape silver nanoparticles at close and far proximity. 
nological Research council of Turkey (TUBITAK).

\section{References}

1. Harry A Atwater, Albert Polman (2010) Plasmonics for improved photovoltaic devices. Nature Materials 9: 205-213.

2. Kelly KL, Coronado E, Zhao LL, et al. (2003) The optical properties of metal nanoparticles: The influence of size, shape and dielectric environment. J Phys Chem B 107: 668-677.

3. Mogensen BK, Kneipp K (2014) Size-dependent shifts of plasmon resonance in silver nanoparticle films using controlled dissolution: Monitoring the onset of surface screening effects. J Phys Chem C 48: 28075-28083.

4. Jung HY, Yeo IS, Kim TU, et al. (2018) Surface plasmon resonance effect of silver nanoparticles on a $\mathrm{TiO} 2$ electrode for dyesensitized solar cells. Surface science 432: 266-271.

5. Raza S, Yan W, Martijn Wubs NS, et al. (2013) Blueshift of the surface plasmon resonance in silver nanoparticles: Substrate effects. Optics Express 21: 27344-27355.

6. K R Catchpole, A Polman (2008) Plasmonic solar cells. Optics Express 16: 21793-21800.

7. Ricky BD, Thomas P, Schmidt-Mende L (2012) Highly absorbing solar cells: A survey of plasmonics. Optics Express 20: A177-A189.

8. Alp Artar, Ahmet A Yanik, Hatice Altug (2011) Multispectral Plasmon Induced Transparency in Coupled Meta-Atoms. Nano letters 11: 1685-1689.
9. FA Oskooi, D Roundy, M Ibanescu, et al. (2010) MEEP: A flexible free-software package for electromagnetic simulations by the FDTD method. Computer Physics Communications 181: 687-702.

10. Stephen KG, Teobald K (2003) Propagation of light in metallic nanowire arrays: Finite-difference time-domain studies of silver cylinders. Phys Rev B 68: 045415.

11. Shunping Z, Kui B, Naomi JH, et al. (2011) Substrate-induced fano resonances of a plasmonic nanocube: $A$ route to increasedsensitivity localized surface plasmon resonance sensors revealed. Nano lett 11: 1657-1663.

12. Jesper J, Thomas GP, Thomas S, et al. (2010) Electrostatic plasmon resonances of metal nanospheres in layered geometries. Phys Rev B 81: 125413.

13. Hohenester U, Trügler A (2012) MNPBEM - A Matlab toolbox for the simulation of plasmonic nanoparticles. Computer Physics Communications 183: 370-381.

14. Anthony C, Fang X, Neil A (2011) Light absorption and field enhancement in two-dimensional arrays of closely spaced silver nanoparticles. J Opt Soc Am B 28: 325-330.

15. PB Johnson, RW Christy (1972) Optical constants of the noble metals. Phys Rev B 6: 4370-4379.

16. Anton K, Martin W, Sona F, et al. (2012) Influence of surface oxidation on plasmon resonance in monolayer of gold and silver nanoparticles. J Appl Phys 112: 103531. 